\title{
TORSIÓN TESTICULAR EN EL PACIENTE GERIÁTRICO. Y REVISIÓN DE LA LITERATURA: A PROPÓSITO DE UN CASO
}

\author{
I. GÓMEZ GARCÍA, J.L. RUIZ RUBIO, E. SANZ MAYAYO, S. CONDE SOMESO,
} G. BRIONES MARDONES, A. LINARES QUEVEDO, R. GARCÍA NAVAS, F.J. BURGOS REVILLA, A. ESCUDERO BARRILERO

Servicio de Urología del Hospital Ramón y Cajal. Madrid.

Actas Urol Esp. 27 (6): 465-467, 2003

\section{RESUMEN}

TORSIÓN TESTICULAR EN EL PACIENTE GERIÁTRICO. Y REVISIÓN DE LA LITERATURA: A PROPÓSITO DE UN CASO

La torsión testicular en el adulto, es una nosología infrecuente aunque debe de entrar dentro del diagnostico diferencial de cualquier adulto de mas de treinta años, con cuadro de escroto agudo. El retraso e incorrecto diagnóstico, aumenta la frecuencia de orquiectomías por procesos vasculares en el adulto frente al niño. Presentamos el caso de varón de 74 años con escroto agudo, y revisión de la literatura.

PALABRAS CLAVES: Torsión testicular. Adulto. Escroto agudo.

\section{ABSTRACT}

TESTICULAR TORSION IN ADULTS AND LITERATURE REVISION: A PROPOS OF ONE CASE

Testicular torsion in adult, is an uncomunly etiology, but we should make diferencial diagnostic in every person over thirty years old, with accurate escrotum. The wrong diagnostic gives up the incidence orchiectomy in adults for vascular etiology. We report a male of 74 years old with acurate escrotum andreview ofthe literature

KEY WORD: Testicular torsion. Adult. Acuratte escrotum.

$\mathrm{L}$ a torsión testicular es una afectación frecuente en la infancia, cuya incidencia es de uno por cada 4000 varones. Varía según los autores teniendo dos picos máximos a los 8 y 13 años, disminuyendo progresivamente según avanza la edad. Conforme el hombre va envejeciendo, la etiología del escroto agudo va siendo mayormente infecciosa (orquitis y orquioepididimitis) e infrecuentemente vascular. Aunque es anecdótico, la torsión testicular en el adulto, existe; por lo tanto siempre debe formar parte del diagnostico diferencial del escroto agudo en mayores de 30 años. Un rápido diagnóstico y tratamiento aumentan la viabilidad del testículo, y disminuyen la probabilidad de orquiectomía

\section{CASO CLÍ́NICO}

Varón de 74 años de edad que acude al servicio de urgencia de nuestro hospital hacia las 4:00 de la mañana, con dolor intenso en hemiescroto izquierdo, que se irradia a hipogastrio, fosa ilíaca y zona lumbar izquierda, el paciente presenta un intenso cortejo vegetativo, con nauseas y vómitos e intenso dolor en teste izquierdo, hipogastrio y 
fosa ilíaca izquierda. El dolor apareció de manera súbita y aumentaba progresivamente de intensidad, a pesar del tratamiento con opiáceos. No presentaba fiebre, ni clínica miccional, tampoco refería alteraciones del ritmo intestinal. Se diagnostica de crisis renoureteral izquierda, siendo tratada como tal y ante la ausencia de mejoría del cuadro se solicita valoración a las tres horas por parte del servicio de urología.

\section{a) Antecedentes personales:}

Como antecedentes de interés el paciente refiere una cardiopatía severa con dos episodios de infarto agudo de miocardio, y angor inestable. También presenta enfermedad pulmonar obstructiva crónica (con intenso componente obstructivo) y dos accidentes isquémicos transitorios. Preguntado por antecedentes urológicos no refería hematurias, ni crisis renoureterales, tampoco había tenido episodios previos de infecciones del tracto urinario.

\section{b) Exploración física:}

Constantes eran normales. En la exploración torácica destacaba disminución de la ventilación en ambos campos inferiores. Abdomen blando y depresible, sin signos de irritación peritoneal. No se palpan masas ni megalias, ni tampoco hernias. Discreto dolor a la percusión en fosa ilíaca izquierda la puño-percusión renal bilateral negativa.

Exploración genital, pene y el teste derecho absolutamente normales. El teste izquierdo se encontraba horizontalizado y ascendido hasta orificio inguinal superficial, y muy doloroso a la palpación. El reflejo cremastérico abolido. El signo de Prehn era positivo. No existían alteraciones inflamatorias en la piel del escroto.

\section{c) Pruebas complementarias:}

Sedimento urinario de rutina fue informado de normal. Realizamos un estudio preoperatorio, el cual es normal salvo bloqueo de rama derecha que se aprecia en el electrocardiograma.

\section{d) Actitud y tratamiento:}

Tras la reexploración del paciente, la intensa sospecha diagnóstica y la demora en el diagnóstico decidimos realizar exploración testicular vía escrotal, en la cual apreciamos torsión del cordón espermático de $180^{\circ}$ y teste izquierdo congestivo e isquémico; destorsionamos, y aplicamos suero fisiológico caliente y a los 10 minutos el testículo volvió a recuperar su tonalidad normal, con posterior pexia bilateral. Dos días después de la intervención el paciente fue dado de alta.

\section{DISCUSIÓN}

La torsión testicular es una etiología frecuente de escroto agudo en la infancia, con una incidencia aproximada de 1 por cada 4000 varones menores de 25 años $^{1}$. En el adulto la frecuencia de esta nosología es menor, pero no inexistente, variando según las series entre el $8 \%$ y el $28 \%^{1-3}$. La incidencia disminuye con la edad. Refiriéndose en diversos estudios incidencias del 26 al 39\% en mayores de 21 años que disminuye hasta el 10\% en mayores de 30 años $^{3,4,8}$.

Debido a la rareza de esta patología en adultos, y la mayor prevalencia de procesos infecciosos e inflamatorios en varones de más de 30 años, como causa de escroto agudo, muchos de estos casos no son diagnosticados o incluso son etiquetados erróneamente y tratados como procesos infecciosos ${ }^{7}$.

En un estudio realizado por Whiterington y colaboradores $^{8}$, el 69\% de los casos de torsión en el adulto fue en el lado izquierdo, y un $31 \%$ en el lado derecho.

La mayor parte de los casos de torsión en el adulto suelen ser intravaginales ${ }^{7}$, aunque también se han descrito no más de 5 casos de torsión extravaginal ${ }^{10-12}$ (forma muy común en lactantes y neonatos). En la literatura se han recogidos casos torsión testicular bilateral $\operatorname{con}^{13} \mathrm{y}$ sin traumatismo previo ${ }^{14,15}$.

El diagnóstico tardío, la creencia errónea de lo inusual de la torsión en el adulto, hace que la tasa de orquiectomía en el adulto sea de un $75 \%$ frente a un 20 a 50\% en niños ${ }^{5,6}$.

Con el diagnóstico de presunción de torsión testicular, la prueba diagnóstica de elección es la ultrasonografía con doppler, que alcanza una certeza diagnóstica de más del $90 \%{ }^{16}$. La conjunción del doppler con la gammagrafia con tecnecio 99 marcado $\left(\mathrm{Tc}^{99 \mathrm{~m}}\right)$, se correlaciona en un $100 \%$ de los casos con los hallazgos quirúrgicos encontra$\operatorname{dos}^{20}$. En muchos centros la ausencia de dispo- 
nibilidad de estos medios diagnósticos, bien por falta de medios o de personal cualificado durante 24 horas; debe hacernos plantar la exploración quirúrgica ante una intensa sospecha ${ }^{17-19}$.

No sólo el tiempo de evolución es importante para la viabilidad del parénquima testicular, también influye el grado de torsión del cordón. Así se han documentado cambios histológicos del parénquima testicular, a las seis horas del inicio del dolor ${ }^{21}$. Sonda y Lapides ${ }^{9}$, en estudios experimentales con perros, en los que se le realizo una torsión de $1080^{\circ}$ la viabilidad del testículo quedo reducida a menos de dos horas.

\section{CONCLUSIONES}

La torsión testicular es una patología presente en el adulto. En la mayoría de los casos es erróneamente diagnosticada de proceso infeccioso y tratado como tal. La edad no debe ser criterio de exclusión en esta patología, y ante la sospecha debemos actuar de igual manera que ante un varón joven, y evitar que la incidencia de orquiectomía en el adulto sea del $75 \%$.

\section{REFERENCIAS}

1. WILLIAMSON RC.: Torsion of the testis and allied conditions. Brit J Surg 1976; 63: 465.

2. CHAPMAN RN, WALTON AJ.: Torsion of the testis and its appendages. Brit Med $J$ 1972; 1: 164.

3. LEE LM, WRIGHT JE.: Testicular torsion in the adult. J Urol 1983; 130: 93.

4. WHITERINGTON R, JARREL TS.: Torsion of the sprematic cords in adult. J Urol 1990; 143: 62.

5. PERRY S, HOOPINGAM D.: Testicular torsion in the older patient. Ann Emerg Med 1983; 12: 319.
6. ALTAFFER LF.: Testicular torsion in men. $J$ Urol 1985; 123: 37-38.

7. THOMAS A.: Testicular torsion in a 59 years old man. J Urol 1995; 154: 1480.

8. WHITERINGTON R, JARREL TS.: Torsion of the spermatic cord in adult. J Urol 1990; 143: 62.

9. SONDA LP, LAPIDES J.: Experimental torsion of the spermatic cord. Surg Forum 1961; 12: 502.

10. JASON C, ABBEY MD.: Extravaginal torsion of spermatic cord in adult. Urology 1991; 38: 79.

11. BAKER K.: Torsion of the testis. Br J Urol 1964; 36 : 35.

12. FARLAND MC.: Testicular estrangulaton in children. Br J Surg 1966; 53: 110.

13. SHEFI S.: Simoultaneus bilateral torsion in an adult. J Urol 1996; 159: 206-207.

14. KOSSOW AS.: Bilateral synchronus testicular torsion, a case report. $J$ Urol 1994; 152: 1.211.

15. WHASNICK RJ.: Simultaneous bilateral torsion of the testes in a man. J Urol 1981; 125: 427.

16. PATRIQUIN HB.: Testicular torsion in infants and children: diagnosis whith doppler sonography. Radiology 1983; 188: 781.

17. BREWER M.: Adult testicular torsion. Urology 1986; 27 (4): 306.

18. Wright JE.: Torsion of the testis. $B r J$ Surg 1996; 64: 274-276.

19. CASS AS.: Elective ochydopexy for recurrent testicule torsion. J Urol 1983; 127: 253-254.

20. RODRIGGEZ DD.: Doppler ultrasounds versus sacaning in the evaluation of the acurate scrotum. J Urol 1981; 125: 343-346.

21. SMITH G.: Cellular changes from graded testicular ischemia. J Urol 1955; 73: 355.

Dr. I. Gómez García

C/ Costa Brava, 9 - Portal 3, 1ํ D

28034 Madrid

(Trabajo recibido el 23 octubre de 2002) 